\title{
Successful outcome of disseminated Candida tropicalis osteomyelitis on remission induction for childhood Philadelphia chromosome-positive acute lymphoblastic leukaemia-case report
}

Lichun Xie ${ }^{1,2}$, Qingling Long ${ }^{2}$, Guichi Zhou ${ }^{2}$, Sixi Liu ${ }^{2}$ and Fei-Qiu Wen ${ }^{1,2^{*}}$ (D)

\begin{abstract}
Background: Invasive fungal infection (IFI) is one of the most challenging complications in children undergoing acute lymphoblastic leukaemia (ALL) treatment, but acute fungal osteomyelitis $(\mathrm{OM}$ ) is rarely encountered.
\end{abstract}

Case presentation: Here, we describe a case of Candida tropicalis osteomyelitis in a 10-year-old patient with Philadelphia chromosome (Ph)-positive ALL. He was on remission induction therapy at the time of neutropenia, and an abscess developed in his right arm. The blood and bone cultures were positive for $C$. tropicalis. Antibiotics and antifungals were administered. Magnetic resonance imaging of the arm revealed an intraosseous abscess, suggestive of OM. Surgical irrigation and debridement of the bone were performed immediately. The patient was effectively treated with antifungal therapy and ALL treatment. He has fully recovered into complete clinical remission but with visible sequelae on magnetic resonance imaging (MRI). He took oral posaconazole for consolidation until disappearance of the lesion shadows on MRI and received subsequent cycles of chemotherapy in parallel.

Conclusions: In the successful management of Ph-positive ALL, dasatinib, a second-generation Abl-tyrosine kinase inhibitor, is crucial. The recommended treatment for Candida osteomyelitis in Ph-positive ALL patients is a fungicidal agent combined with surgery and modification chemotherapy with dasatinib. The use of combined modalities of treatment seems to be crucial in the successful management of Ph-positive ALL.

Keywords: Candida tropicalis, Case report, Dasatinib, Immunocompromised, Osteomyelitis, Philadelphia chromosome-positive acute lymphoblastic leukaemia

\footnotetext{
* Correspondence: fwen62@163.com

'The First Affiliated Hospital, Jinan University, Guangzhou, Guangdong, China ${ }^{2}$ Department of Hematology/Oncology, Shenzhen Children's Hospital, No.

7019 Yitian Rd, Shenzhen, Guangdong, China
}

(c) The Author(s). 2021 Open Access This article is licensed under a Creative Commons Attribution 4.0 International License, which permits use, sharing, adaptation, distribution and reproduction in any medium or format, as long as you give appropriate credit to the original author(s) and the source, provide a link to the Creative Commons licence, and indicate if changes were made. The images or other third party material in this article are included in the article's Creative Commons licence, unless indicated otherwise in a credit line to the material. If material is not included in the article's Creative Commons licence and your intended use is not permitted by statutory regulation or exceeds the permitted use, you will need to obtain permission directly from the copyright holder. To view a copy of this licence, visit http://creativecommons.org/licenses/by/4.0/ The Creative Commons Public Domain Dedication waiver (http://creativecommons.org/publicdomain/zero/1.0/) applies to the data made available in this article, unless otherwise stated in a credit line to the data. 


\section{Background}

Acute lymphoblastic leukaemia (ALL) is the most common childhood malignancy [1]. Children with ALL are immunocompromised patients who are at high risk of infection associated with high morbidity and mortality rates [2]. Invasive fungal infection (IFI) is one of the most challenging complications in children with ALL treatment [3], but acute fungal osteomyelitis (OM) is rarely encountered. In immunocompromised children, the most common mechanism of these complications includes fungal osteomyelitis due to continuous infiltration or haematogenous spread [4]. Candida species are weak pathogens that are common commensal organisms inhabiting the skin and mucous membranes of most individuals. However, the rate of incidence of invasive Candida infection is increasing among newborns in the ICU and in immunosuppressive therapy, the augmented use of intravenous catheters and haematological malignancy and neutropenia in children [5]. Successfully managing fungal osteomyelitis and chemotherapy for ALL is quite difficult. Here, we report a case of childhood Philadelphia chromosome $(\mathrm{Ph})$-positive acute lymphoblastic leukaemia, complicated by disseminated Candida tropicalis osteomyelitis of the right humerus during remission induction that was successfully managed by timely and appropriate administration without compromising antileukaemia therapy.

\section{Case presentation}

A 10-year-old boy was confirmed to have Philadelphia chromosome (Ph)-positive ALL. According to the Chinese Children's Cancer Group study ALL-2015 (CCCGALL-2015), he was provisionally assigned as intermediate-risk based on his presenting clinical features, aged 10 years, and immunophenotype B- cell and Philadelphia chromosome-positive ALL. According to CCCG-ALL-2015, the patient will receive MRD directed, risk-stratified treatment, modified from the St Jude Children's Research Hospital Total XV and XVI studies and the Shanghai Children's Medical Center ALL-2005 trial. For $M R D \geq 1 \%$ at the end of remission induction, provisional intermediate-risk patient has to be escalated to high risk. Allogeneic transplant was recommended. He received upfront window therapy with dexamethasone for 4 days followed by remission induction with prednisone acetate, vincristine, daunorubicin hydrochloride (DNR), and pegaspargase from days 5 to 28 .

$\mathrm{He}$ began to receive dasatinib $\left(80 \mathrm{mg} / \mathrm{m}^{2}\right.$ per day $)$ as soon as the diagnosis was made on day 5 of remission induction. On day 18 of remission induction, he was hospitalized for cough, fever, and pain, tenderness and swelling over the right elbow. Laboratory tests were notable for neutropenia and elevated inflammatory markers (Fig. 1). A computed tomography (CT) chest scan showed the patchy shadows in the anterior lobe of left

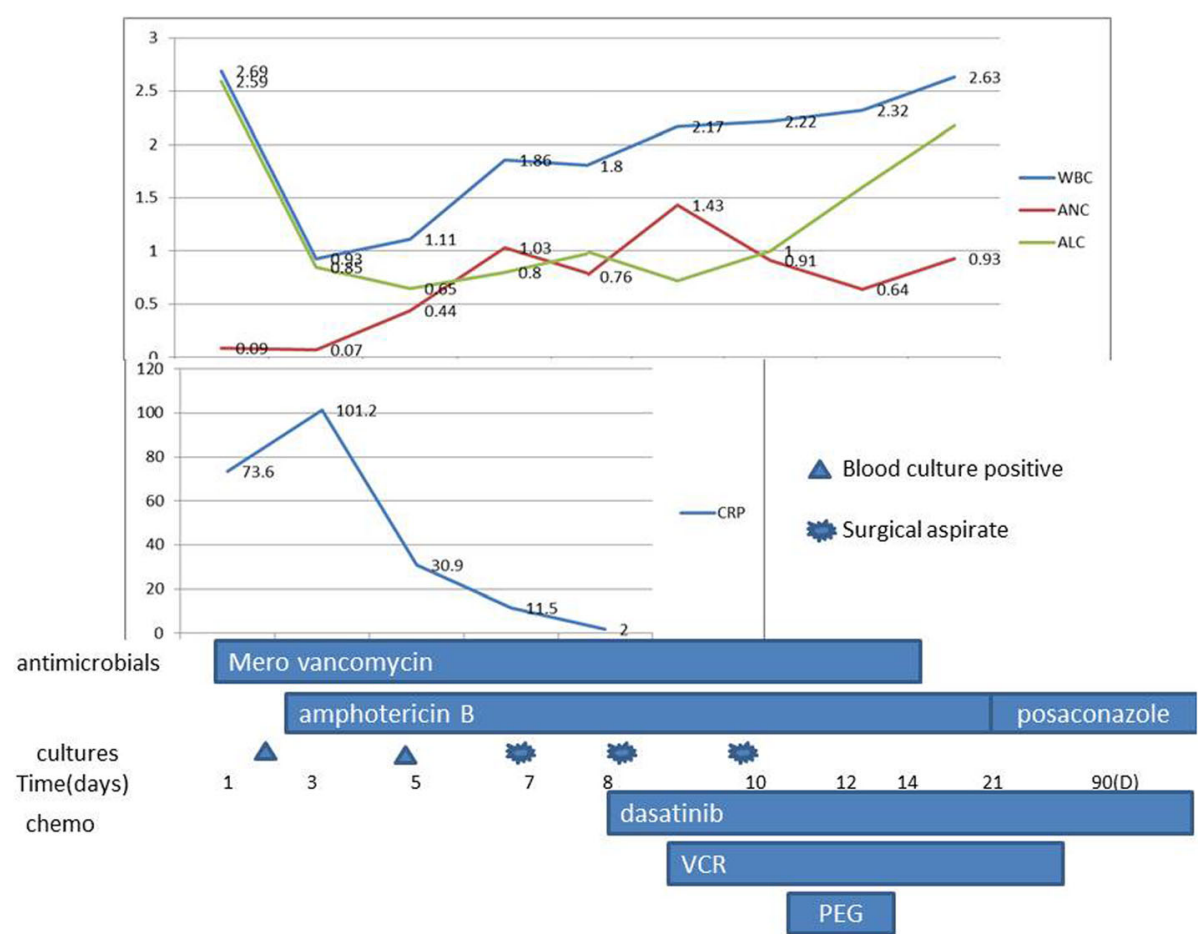

Fig. 1 The results of the laboratory test, antimicrobial treatment and chemotherapy 
upper lung and the middle lobe of right lung. The right upper limb ultrasound revealed swelling of soft tissue without previous trauma. Empiric treatment with broadspectrum antibiotics was initiated to cover both grampositive and gram-negative bacteria. The lesion progressed to an abscess in the upper right arm. Twentytwo hours later, blood culture showed fungus positivity and pus. Empirical amphotericin B therapy was initiated. There are two reasons we chose amphotericin B. First, Candida infection is the most common fungal infection. Second, vincristine is one of the drugs used in remission induction chemotherapy. Voriconazole may increase the toxic side effects of vincristine, so voriconazole was not our first option [6]. The blood culture results suggested C. tropicalis 2 days later. Minimal inhibitory concentrations indicated susceptibility to amphotericin B (minimal inhibitory concentration $<=0.5 \mu \mathrm{g} / \mathrm{mL}$ ), fluconazole (1) and voriconazole $(0.125)$ and flucytosine $(<=0.4)$. He subsequently developed pain and swelling over the right upper arm and forearm. Five days after admission, he was afebrile, but pain, oedema, and limited function of the right elbow were progressive. Repeated ultrasound revealed discrete periosteal oedema adjacent to the humerus 1 week after admission. Magnetic resonance imaging (MRI) of the right humerus showed an

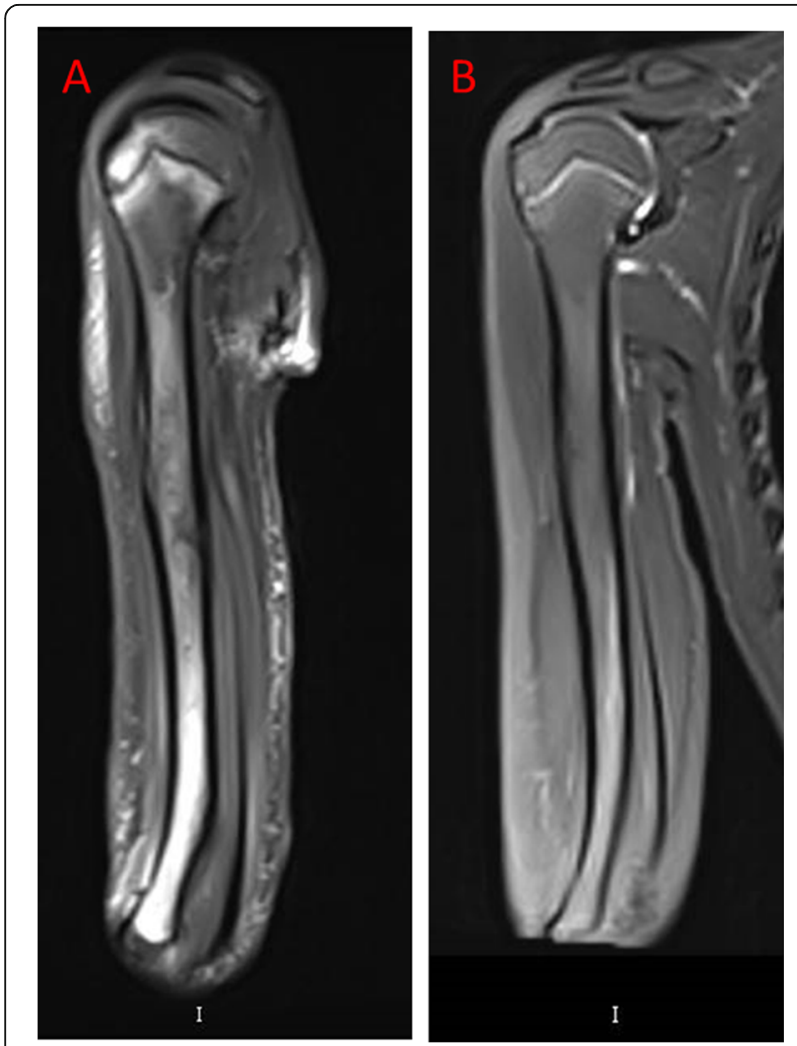

Fig. 2 The MRI results of the right arm at first (a). At the follow-up MRI, the previous abnormal signals narrowed significantly (b) intraosseous abscess and probable osteomyelitis (Fig. 2). Surgical irrigation and debridement of the bone were performed immediately (Fig. 3). Culture of bone aspirates obtained during surgery showed that $C$. tropicalis was sensitive to amphotericin $B$, which is the same result as the blood culture. A small study of the bone fragments collected during surgery revealed fungal spores. All chemotherapy, except prednisone was continued, was withdrawn for 1 week. Debridement was repeated on the third postoperative day, resulting in considerable improvement of clinical and laboratory findings. His condition improved. One week later, when he was nontoxic, haemodynamically stable, with no signs of haematopoietic toxicity and inflammatory markers within the normal range, induction chemotherapy was continued with some modification with prednisolone and dasatinib, and treatment was carried out for osteomyelitis with amphotericin B, meropenem and vancomycin in parallel. Successful control of osteomyelitis was achieved by serial surgical debridement of the lesion, antibiotics, amphotericin $\mathrm{B}$ and $\mathrm{HBO}$ therapy. Treatment with amphotericin B was administered for a total of 3 weeks; during this time, he regained the ability to move his arm and transitioned to oral posaconazole for consolidation, which he is currently receiving on an ongoing basis. Vincristine (D19 and D26) and L-asparaginase (D26) were administered until the fever was controlled and the neutrophil

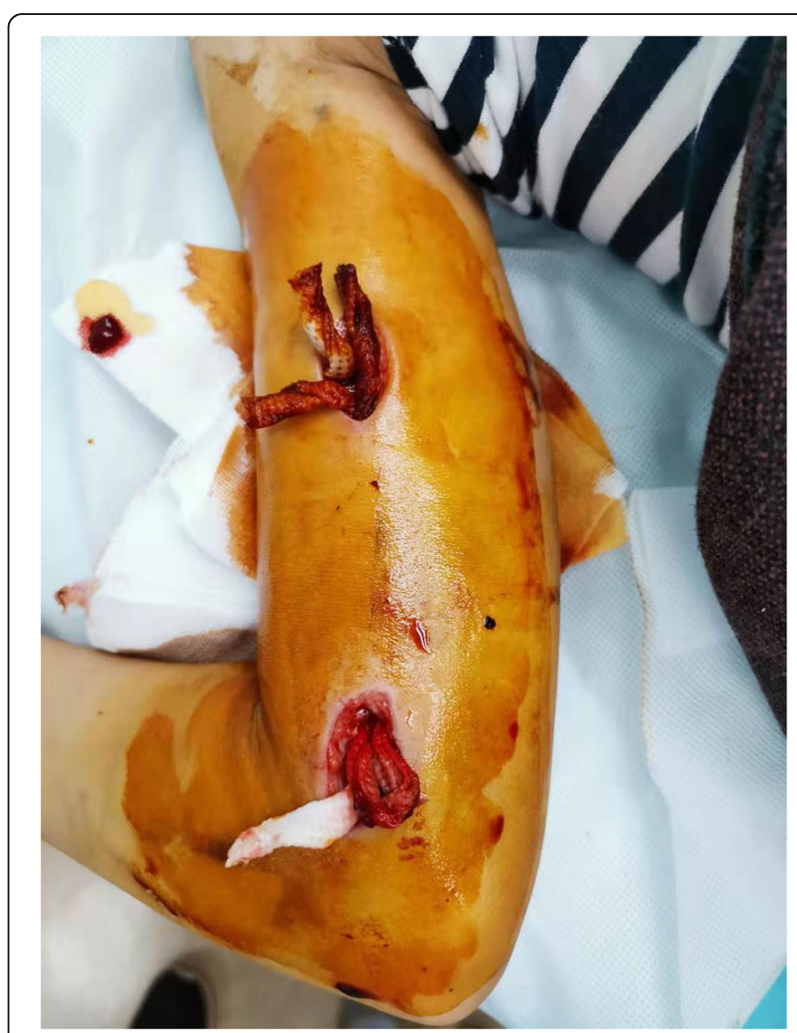

Fig. 3 Surgical irrigation and debridement of the bone 
count (ANC) exceeded $500 / \mathrm{mm}^{3}$. After completion of remission induction therapy, he achieved complete remission (CR). Then, we removed the remission induction treatment consisting of cyclophosphamide (CTX), cytarabine (Ara-c), and mercaptopurine (6-MP) from days 29 to 35 . The next chemotherapy regimen was continued with dasatinib, weekly vincristine and daily mercaptopurine for 3 weeks. Subsequent cycles of chemotherapy were reinitiated as per the protocol. At follow-up 5 months later, the patient continued to feel good.

\section{Discussion and conclusions}

Candida are found on the skin and in the respiratory tract, vagina and stools. According to a multicentre study in Australia [7], Candida species accounted for the majority of infections associated with neutropenia in immunocompromised patients with ALL, of which Candida albicans and Candida parapsilosis were most frequently detected. According to another retrospective study, the proportion of Candida is increasing in children with ALL [8]. To our knowledge, there are few reports of C. tropicalis osteomyelitis in a child with ALL. Predisposing factors present in our case were underlying leukaemia, steroids, neutropenia, and immunosuppressive therapy, which is consistent with the literature report [9].

Proving a diagnosis is the first key step in the successful management of IFI. Isolating and identifying the causative fungus on culture is important for epidemiological and research purposes. The culture of specimens from surgery is more sufficient than blood to determine the pathogen and helps to establish the diagnosis as quickly as possible.

A combination of early diagnosis, surgical debridement and antifungal therapy seems to be crucial. The timely administration of empirical amphotericin B on suspicion of fungal infection in our case along with debridement of necrotic tissue may be responsible for a successful outcome.

In the successful management of Ph-positive ALL, in the pre-TKI era, matched donor allogeneic stem cell transplantation was beneficial [10]. Sequential addition of imatinib (a tyrosine kinase inhibitor) to the chemotherapy regimen in Ph-positive ALL dramatically improved the outcomes for children with Ph-positive ALL [11]. TKIs administered in the early phases of therapy can dramatically improve the outcome of childhood $\mathrm{Ph}$ positive ALL. According to the study, there was no significant difference in 5-year disease-free survival (DFS) for patients who received imatinib alone versus patients who received imatinib followed by HSCT [12]. Dasatinib is a crucial second-generation Abl-tyrosine kinase inhibitor. According to our randomized clinical trial, dasatinib at a dosage of $80 \mathrm{mg} / \mathrm{m}^{2}$ per day yielded superior results in the treatment of Ph-positive ALL compared with imatinib mesylate at a dosage of $300 \mathrm{mg} / \mathrm{m} 2$ per day [13]. Anti-infection combined with surgical incision and drainage and dasatinib administration together led to the successful treatment of osteomyelitis while maintaining a remission state of Ph-positive ALL.

The importance of judicious concomitant administration of antifungal and anti-leukaemia treatment during induction therapy in ALL is highlighted. Moreover, we emphasize that the most efficient diagnostic and treatment strategy for patients with fungal osteomyelitis with ALL is the combination of medical and surgical management. When there is significant marrow toxicity and infection in a patient with Ph-positive ALL, concurrent myelosuppressive agents such as DNR, Ara-C, CTX, MTX, and 6-MP should be stopped instead of dasatinib. It can be an effective treatment option for patients who need both infection control and ALL treatment.

\section{Abbreviations \\ ALL: Acute lymphoblastic leukaemia; Ara-c: Cytarabine; CCCG-ALL- 2015: Chinese Children's Cancer Group study ALL-2015; CT: Computed tomography; CR: Complete remission; CTX: Cyclophosphamide; DNR: Daunorubicin hydrochloride; IFI: Invasive fungal infection; OM: Osteomyelitis; Ph: Philadelphia chromosome; ANC: Neutrophil granulocyte; MRI: Magnetic resonance imaging; 6-MP: Mercaptopurine}

\section{Acknowledgements}

Sanming Project of Medicine in Shenzhen (SZSM201512033).

\section{Authors' contributions}

XLC designed the data analysis and wrote the manuscript; Qingling Long and Guichi Zhou provided imaging assistance; Sixi Liu and Feiqiu Wen contributed to critical revision. All of the authors have read and approved the manuscript.

\section{Funding}

Not applicable.

\section{Availability of data and materials}

The microarray data will be available in the NCBI database.

\section{Ethics approval and consent to participate}

This study was approved by the ethics committee of Shenzhen Children's Hospital. All procedures performed in studies involving human participants were in accordance with the ethical standards of the institutional and/or national research committee and with the 1964 Helsinki Declaration and its later amendments or comparable ethical standards.

\section{Consent for publication}

Written consent to publish this information was obtained from the parents of the study participants.

\section{Competing interests}

The authors declare that they have no competing interests.

Received: 16 July 2020 Accepted: 14 December 2020

Published online: 11 February 2021

\section{References}

1. Hunger SP, Mullighan CG. Acute lymphoblastic leukemia in children. N Engl J Med. 2015;373:1541-52. 
2. Santiago R, Vairy S, Sinnett D, Krajinovic M, Bittencourt H. Novel therapy for childhood acute lymphoblastic leukemia. Expert Opin Pharmacother. 2017; 18:1081-99.

3. Inaba H, Pei D, Wolf J, Howard SC, Hayden RT, Go M, et al. Infection-related complications during treatment for childhood acute lymphoblastic leukemia. Ann Oncol. 2017;28:386-92.

4. Sahbudak Bal Z, Yilmaz Karapinar D, Karadas N, Sen S, Onder Sivis Z, Akinci $A B$, et al. Proven and probable invasive fungal infections in children with acute lymphoblastic leukaemia: results from an university hospital, 20052013. Mycoses. 2015;58:225-32.

5. Wisplinghoff $H$, Seifert $H$, Tallent SM, Bischoff T, Wenzel RP, Edmond MB Nosocomial bloodstream infections in pediatric patients in United States hospitals: epidemiology, clinical features and susceptibilities. Pediatr Infect Dis J. 2003;22:686-91.

6. Moriyama B, Henning SA, Leung J, Falade-Nwulia O, Jarosinski P, Penzak SR, et al. Adverse interactions between antifungal azoles and vincristine: review and analysis of cases. Mycoses. 2012;55:290-7.

7. Wang SS, Kotecha RS, Bernard A, Blyth CC, McMullan BJ, Cann MP, et al. Invasive fungal infections in children with acute lymphoblastic leukaemia: results from four Australian centres, 2003-2013. Pediatr Blood Cancer. 2019; 66:e27915.

8. Cesaro S, Tridello G, Castagnola E, Calore E, Carraro F, Mariotti I, et al. Retrospective study on the incidence and outcome of proven and probable invasive fungal infections in high-risk pediatric onco-hematological patients. Eur J Haematol. 2017;99:240-8.

9. Bartlett AW, Cann MP, Yeoh DK, Bernard A, Ryan AL, Blyth CC, et al. Epidemiology of invasive fungal infections in immunocompromised children; an Australian national 10-year review. Pediatr Blood Cancer. 2019. 66:e27564.

10. Aricò M, Schrappe M, Hunger SP, Carroll WL, Conter V, Galimberti S, et al. Clinical outcome of children with newly diagnosed Philadelphia chromosome-positive acute lymphoblastic leukemia treated between 1995 and 2005. J Clin Oncol. 2010;28:4755-61.

11. Schultz KR, Bowman WP, Aledo A, Slayton WB, Sather H, Devidas M, et al. Improved early event-free survival with imatinib in Philadelphia chromosome-positive acute lymphoblastic leukemia: a children's oncology group study. J Clin Oncol. 2009;27:5175-81.

12. Schultz KR, Carroll A, Heerema NA, Bowman WP, Aledo A, Slayton WB, et al. Long-term follow-up of imatinib in pediatric Philadelphia chromosomepositive acute lymphoblastic leukemia: children's oncology group study AALL0031. Leukemia. 2014;28:1467-71.

13. Shen S, Chen X, Cai J, Yu J, Gao J, Hu S, et al. Effect of dasatinib vs imatinib in the treatment of pediatric Philadelphia chromosome-positive acute lymphoblastic leukemia: a randomized clinical trial. JAMA Oncol. 2020;6: 358-66.

\section{Publisher's Note}

Springer Nature remains neutral with regard to jurisdictional claims in published maps and institutional affiliations.

Ready to submit your research? Choose BMC and benefit from:

- fast, convenient online submission

- thorough peer review by experienced researchers in your field

- rapid publication on acceptance

- support for research data, including large and complex data types

- gold Open Access which fosters wider collaboration and increased citations

- maximum visibility for your research: over $100 \mathrm{M}$ website views per year

At $\mathrm{BMC}$, research is always in progress.

Learn more biomedcentral.com/submissions 